\title{
Development of Frontward Sensing Radar Using Electromagnetic Waves for the Microtunneling Method
}

\author{
NTT Access Service Systems Laboratories \\ 1-7-1 Hanabatake, Tsukuba City, Ibaraki 305-0805 \\ s223@ansl.ntt.co.jp
}

Shinobu TSUTSUMI, Kyoichi MIYAZAKI, Shigeru YAMAGUCHI, Hidenori HINO

\begin{abstract}
For safe and efficient execution using the microtunneling method, it is necessary to understand both buried objects and ground information. The authors adopted an electromagnetic wave sensing system, devised an antenna that can be installed in a microtunneling driving machine with a diameter of approximately $\varnothing 350 \mathrm{~mm}$, and incorporated an original analysis algorithm to develop a frontward sensing system capable of automatically judging the locations of buried objects. This system made it possible to accurately detect both the presence and distance to buried objects to the front and sides of the driving machine, thus improving driving safety and efficiency.
\end{abstract}

Keywords: frontward sensing, radar, utility detection, electromagnetic wave, microtunneling

\section{INTRODUCTION}

NTT independently developed and marketed the 'ACEMOLE' as a technology for laying electronic communications conduits. The applications of this method have expanded from communications conduit laying to take in installation of underground lifeline such as sewage and water supply pipes and electric cables, and the method has now been used to lay in excess of $700 \mathrm{~km}$ of pipe and conduit.For safe and efficient execution using the microtunneling method, it is necessary to understand both buried objects and ground information. Thus far these investigations have consisted of advance test boring, checking past facility records and drawings, and using equipment for detecting buried objects from above ground. However, these methods have various problems such as discontinuous data and insufficient or unclear data that required follow-up surveys, etc. Therefore, various frontward sensing techniques which detect buried objects by transmitting and receiving elastic waves and ultrasonic waves from antennas or other devices installed in the driving machine have been proposed to solve these problems, and some of these have been applied in the field. However, these technologies were designed for large-bore shield machines, and application to the microtunneling method faces problems such as limits to installation in driving machines and drops in recognition performance due to reduced device sizes. Determining the presence and location of buried objects from the detection data also requires experience and skill, so application is still impractical. Therefore, the authors adopted an electromagnetic wave sensing system, and devised an antenna that can be installed in a microtunneling driving machine with a diameter of approximately $\varnothing 300 \mathrm{~mm}$. An original analysis algorithm for the data obtained from this antenna was also incorporated to develop a frontward sensing system capable of automatically judging the locations of buried objects. This system made it possible to accurately detect both the presence and distance to buried objects to the front and sides of the driving machine.

\section{FRONTWARD SENSING RADER SYSTEM}

The developed frontward sensing radar system is comprised of a radar antenna unit that senses buried objects and a main unit that processes and displays the signals received from the antenna. (Fig. 1) 


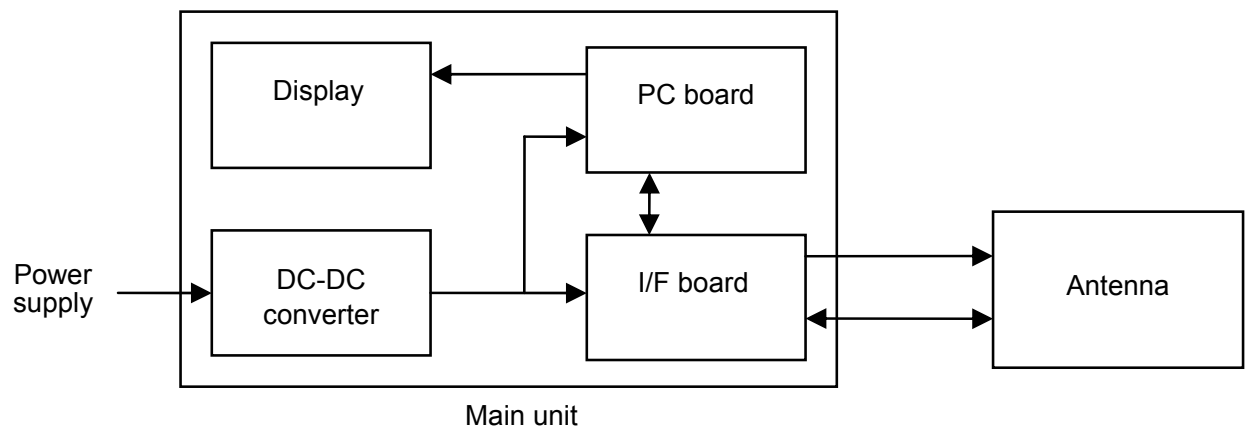

Figure 1. System Configuration

Operators perform driving work while watching the image appearing in the display. In addition, the main unit incorporates an algorithm as described in section 2.3, and is equipped with a function that automatically stops driving when the antenna senses a buried object. This prevents collisions with buried objects and other accidents.

\subsection{Antenna Characteristics}

Fig. 2 shows the receiving antenna and transmitting antenna appearance. The antenna elements have teardrop shapes, with the receiving antennas positioned at the top and bottom and the transmitting antenna in the middle. Compact antennas were newly developed to achieve a size capable of mounting in the front of a driving machine. This made it possible to install the antenna in a driving machine with a diameter of approximately $\varnothing 350 \mathrm{~mm}$. Note that this antenna has a sensing range of $1.5 \mathrm{~m}$ in the frontward direction and $1.2 \mathrm{~m}$ in the lateral direction. (Fig. 3)

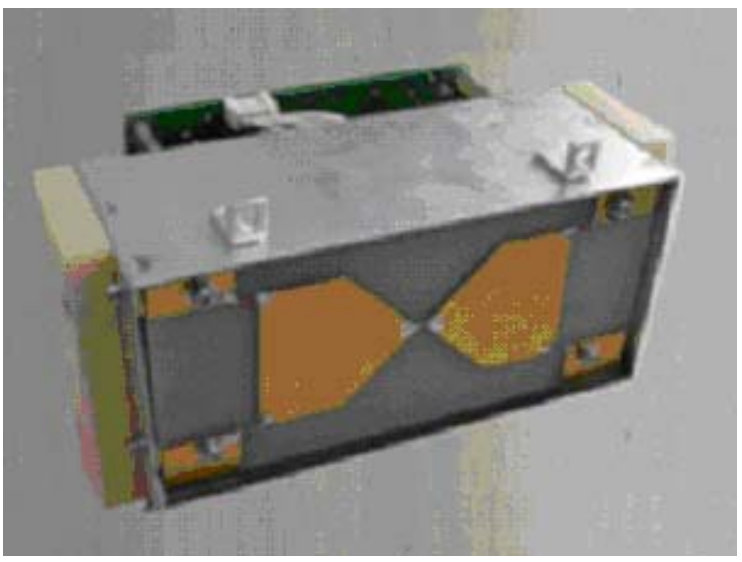

Figure 2. Antenna Unit

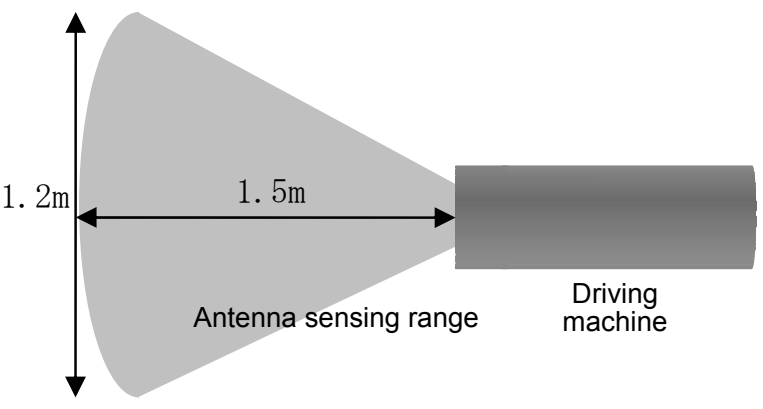

Figure 3. Antenna Sensing Range

\subsection{Antenna Configuration}

The conventional frontward sensing system shown in Fig. 4 could only judge the presence of buried objects, and was incapable of determining the distance to, direction and location of buried objects. Therefore, this new system measures the radio waves from the transmitting antenna with multiple receiving antennas, making it possible to determine the distance to, direction and location of buried objects. Two receiving antennas with offset directional angles are positioned as shown in Fig. 5 so that the upper antenna receives the reflected waves from directly in front and upward, and the lower antenna receives the reflected waves from directly in front and downward. This means that when only the upper antenna catches strong reflected waves, a buried object is present at a front and upward location, and when only the lower antenna catches strong reflected waves, a buried object is present at a front and downward location. Also, when both the upper and lower antennas receive a reflected wave at the same time, a buried object is present in the driving direction. The distance to the buried object is determined by the location judging algorithm described in the following section. 

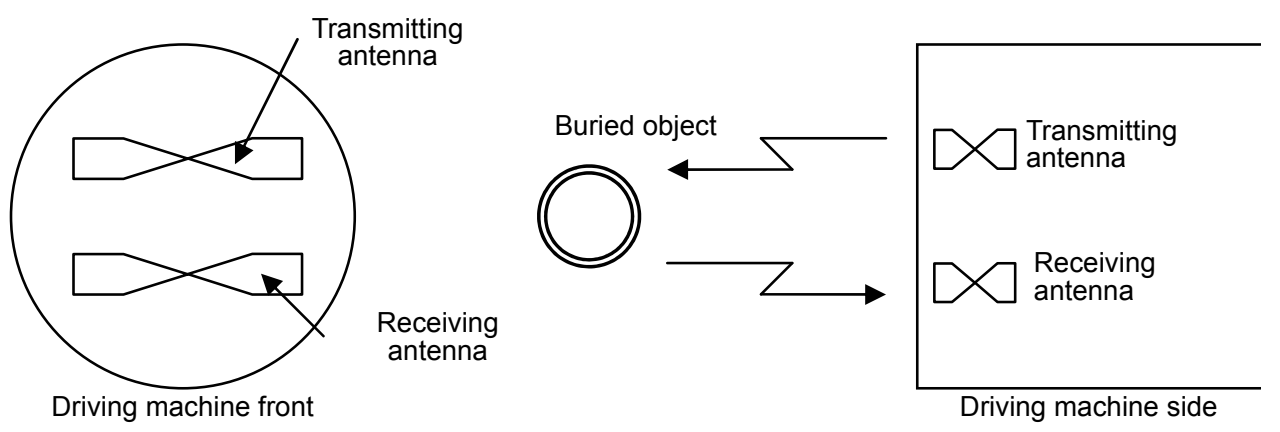

Figure 4. Conventional Antenna Layout

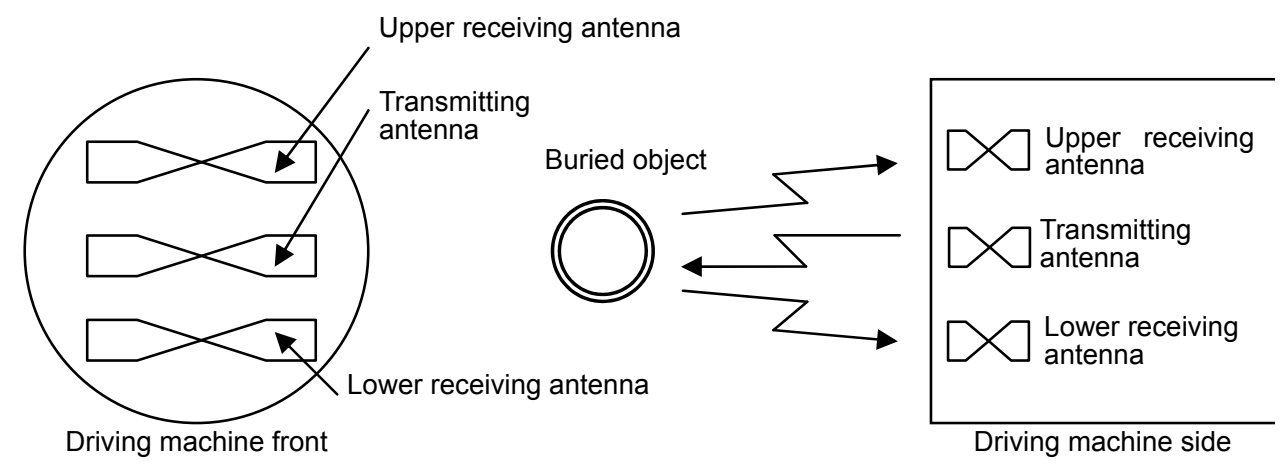

Figure 5. Antenna Layout of this System

\subsection{Location Judging Algorithm}

Fig. 6 shows the algorithm for judging the location of buried objects while driving.

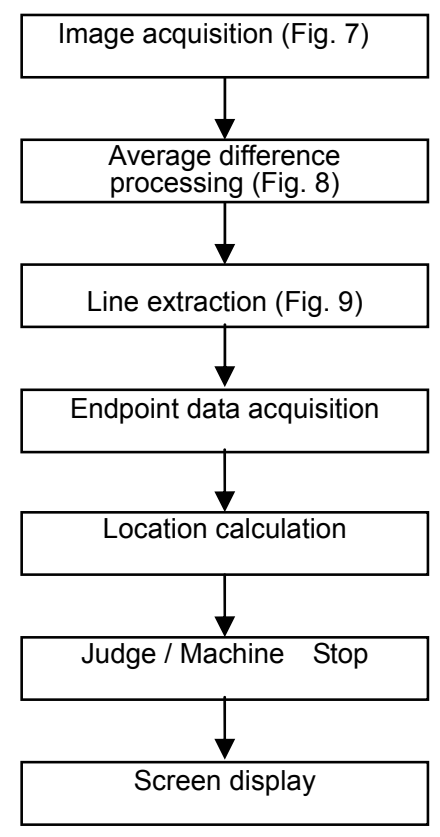

First, the image data from the antenna unit incorporated into the driving machine is obtained online while driving (Fig. 7). Next, average difference processing is performed to eliminate continuous linear reflection in the horizontal direction. (Fig. 8)

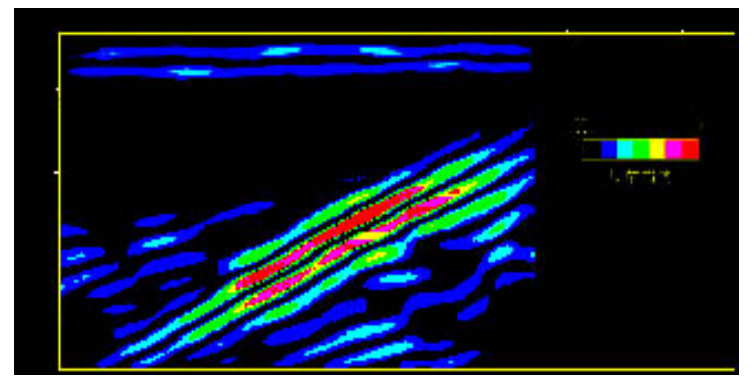

Figure 7. Sensed ImLine extraction

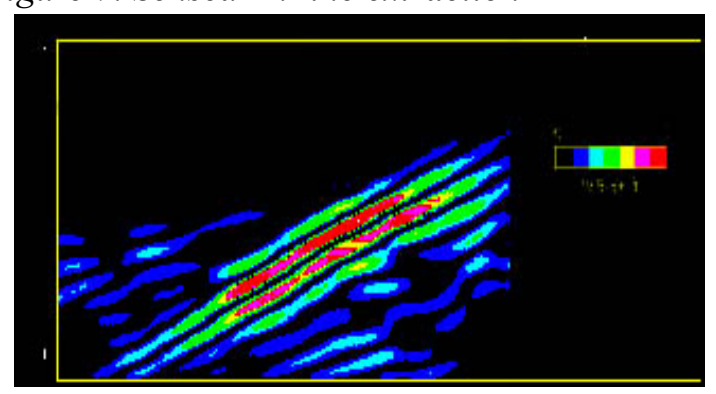

Figure 8. Average Difference Processing

Figure 6. Location Judging Algorithm 


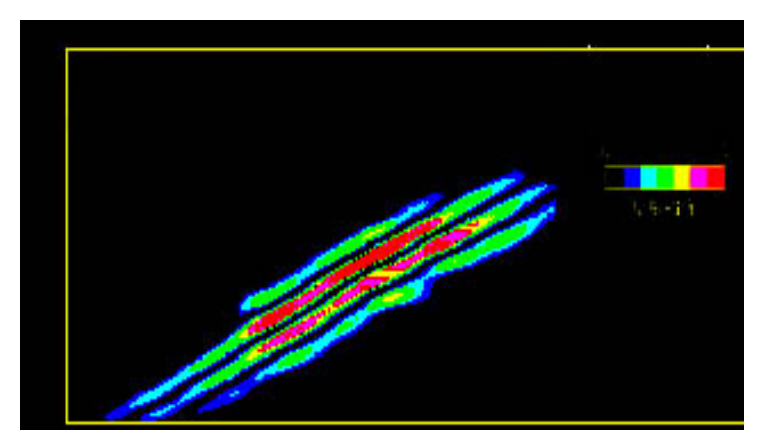

Figure 9. Line Extraction

Then, signals with a reflection strength of a threshold value or higher are extracted from the image remaining after the average difference processing. (Fig. 9) At this time, only the straight lines that satisfy equation (1) are extracted.

$$
A X+B(A \neq 0)
$$

Next, the distance between both endpoints of the extracted lines and the round-trip propagation time of each endpoint are calculated. This distance and round-trip propagation time are then substituted into equation (2) to calculate the distance $\mathrm{L}$ to the obstacle.

$$
L=\frac{c T}{2 \sqrt{\varepsilon_{s}}}
$$

Note that $\mathrm{T}$ is the round-trip propagation time, $\mathrm{c}$ is the speed of light $\left(3 \times 10^{8} \mathrm{~m} / \mathrm{s}\right)$, and $\varepsilon_{s}$ is the dielectric constant which is unique and differs according to the soil quality. The direction, distance and other information concerning the detected buried object are displayed on the screen. Thus, the possibility of a collision is judged from the position information searched for. When there is the possibility of a collision, a machine is stopped automatically.

\section{FUNCTION TESTS}

Basic tests were conducted both above ground and underground using the developed antennas.

\subsection{Basic Above-ground Test}

This test observed changes in the data due to the antenna's advance, and aimed to investigate the beam angle from the transmitting antenna and the sensing range. As shown in Fig. 10, steel pipes for measurement was placed in a wooden pipe position adjustment frame that allowed the pipe position to be adjusted at $10 \mathrm{~cm}$ intervals in the vertical direction. The distance between the antenna and the pipe was then changed, data was obtained, and the angle of antenna beam travel was investigated.

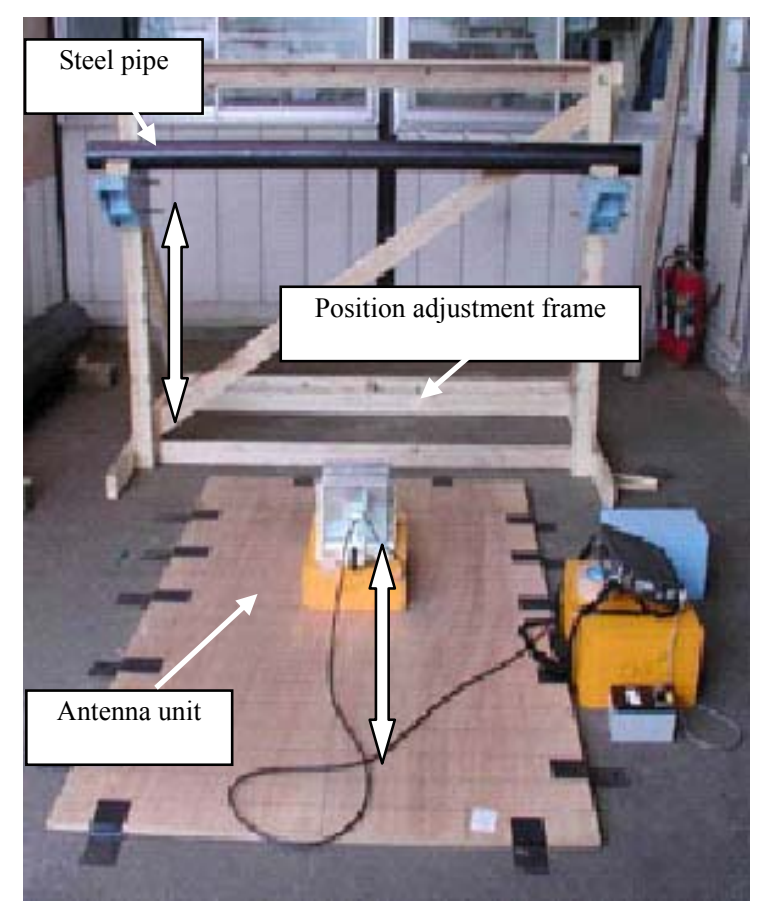

Figure 10. Experimental equipment

\subsection{Above-ground Test Verification Results}

Fig. 11 shows the changes in the center position of the beam. This verification used a steel pipe with a diameter of $\varnothing 100 \mathrm{~mm}$. The results showed that the angle of antenna beam travel changed with an inclination of approximately $45^{\circ}$.

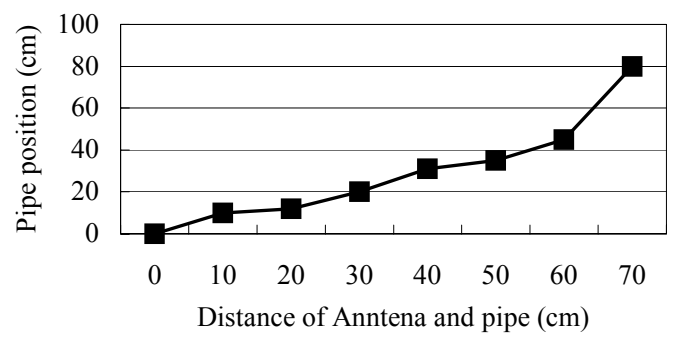

Figure 11. Antenna beam angle

\subsection{Basic Underground Test}

A test soil tank was created with multiple buried pipes (Fig. 12). The reflection characteristics for four buried pipe depths and five patterns of pipe type (steel pipe, vinyl pipe) and pipe inclination 
were investigated with backfill soil conditions of sandy soil and Kanto loam. Data was obtained by placing the antenna in the center of the buried pipe and scanning in the perpendicular direction, and the attenuation characteristics of the electromagnetic waves were investigated.

\subsection{Underground Test Verification Results}

Fig. 13 shows the results of verifying the attenuation characteristics of the electromagnetic waves. The investigation used data with a fixed

gain. As shown by the slopes in the graph, Kanto loam exhibits a greater amount of attenuation than for sandy soil. Also, based on the gain size and the slope for steel pipes at a depth of $0.5 \mathrm{~m}$, the estimated gain at a depth of $0 \mathrm{~m}$ is $35.5 \mathrm{~dB}$ for sandy soil and $20.5 \mathrm{~dB}$ for Kanto loam. This indicates that the reflection is greater when waves are emitted from the antenna into sandy soil than when emitted into Kanto loam. The vinyl pipes showed smaller reflection than steel pipes in both soil qualities, indicating that they have a larger gain. The amount of attenuation in each soil quality is thought not to change according to the pipe type. Based on these results, sensing vinyl pipes at a depth of $1.5 \mathrm{~m}$ requires a gain of $98.5 \mathrm{~dB}$ or more in sandy soil, and $91.5 \mathrm{~dB}$ or more in Kanto loam. This radar system has a gain capability of $100 \mathrm{~dB}$ or more, so it can be applied to these soil qualities.

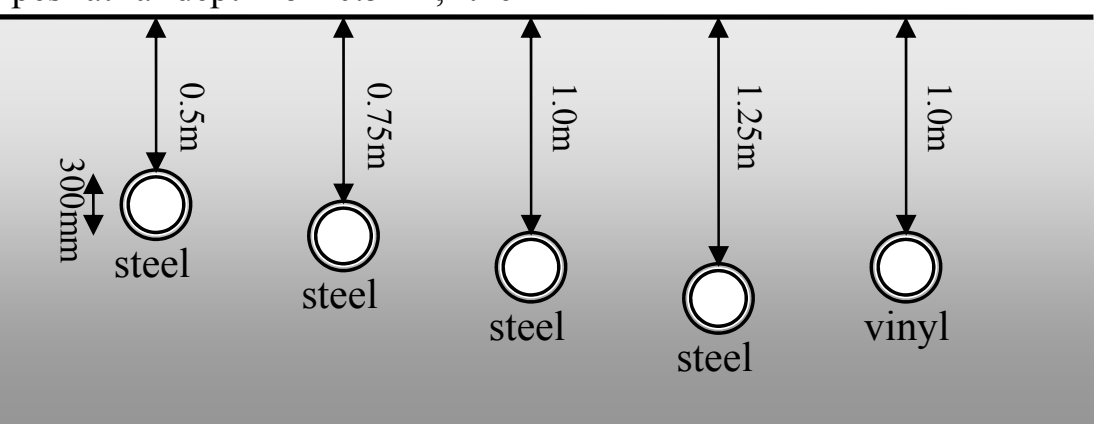

Figure 12. Underground pipe arrengement

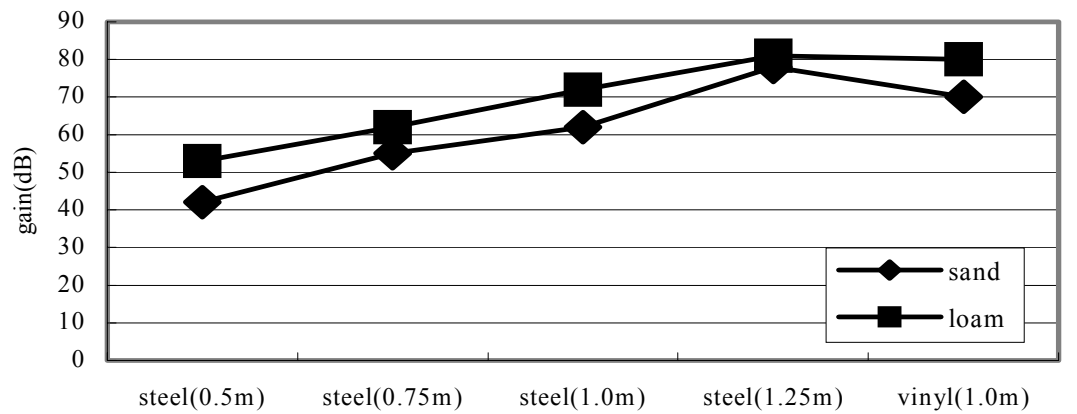

Figure 13. Attenuation characteristics of electromagnetic waves 


\section{CONCLUSION AND FUTURE PLANS}

This paper reported the effectiveness of frontward sensing technology using electromagnetic waves for the microtunneling method. In addition to a newly developed antenna, this system also applied a judgment algorithm to the obtained data to allow automatic judgment of the locations and distance to buried objects.

In the future, driving machine tip materials and man-machine interfaces will be investigated in order to mount the antenna in an actual driving machine. In addition, there are also plans to conduct on-site verification with actual driving machines to further raise the degree of system completeness.

\section{REFFERENCES}

[Miyaratake, Baba, Hara, Sudo and Nagashima] "New ESPAR, the underground radar with great performance and estimation of prospect for NO-DIG investigation in Japan," 18th International Conference on NO-DIG 2000, Perth AUSTRALIA, 2000 ,

[H.Akutsu, J.Masuda, Y.Nagashima, S.Kohno and Y.Sudo]

"Development of Underground Radar System," 8th International Conference on NO-DIG 90, Osaka Japan, pp1-10, 1990,

[Y.Sudo, Y.Nomura and Y.Nagashima]

"Extraction of Cavity Signals from Underground Radar Images Using Frequency Analysis," ICMT2002, pp129-134, 2002

[Teruhisa Awata, Shinobu Tsutsumi, Kyoichi Miyazaki, Shigeru Yamaguchi]

"Development of Frontward Sensing Radar Using Electromagnetic Waves for the Microtunneling Method," NO-DIG2003, WASHINGTON, USA, 2003 